\title{
Partitioning the components of visuomotor adaptation to prism-altered distance
}

Anne-Emmanuelle Priot ${ }^{1,2,3,4}$, Rafael Laboissière ${ }^{2,3,4}$, Justin Plantier ${ }^{1}$, Claude Prablanc ${ }^{2,3,4}$, Corinne Roumes ${ }^{1, *}$

${ }^{1}$ Institut de recherche biomédicale des armées (IRBA), BP 73, 91223 Brétigny-sur-Orge cedex, France

${ }^{2}$ INSERM, U864 Espace et Action, 16 Avenue Lépine, 69676 Bron cedex, France

${ }^{3}$ Biologie Humaine, Université Lyon 1, 69003 Lyon, France

${ }^{4}$ Institut Fédératif des Neurosciences de Lyon, IFR 19, 69003 Lyon, France

${ }^{*}$ Corresponding author:

Corinne Roumes ${ }^{1}$

Institut de recherche biomédicale des armées (IRBA), BP 73, 91223 Brétigny-sur-Orge cedex, France

E-mail: croumes@imassa.fr; Tel.: +33 (0) 1692378 72; Fax: +33 (0) 169238186

E-mail address of each author:

Anne-Emmanuelle Priot: aepriot@imassa.fr

Rafael Laboissière: rafael.laboissiere@inserm.fr

Justin Plantier: jplantier@imassa.fr

Claude Prablanc: claude.prablanc@inserm.fr

Corinne Roumes: croumes@imassa.fr

\begin{abstract}
While the mechanisms of short-term adaptation to prism-altered apparent visual direction have been widely investigated, the processes underlying adaptation to prism-altered perceived distance are less well known. This study used a hand-pointing paradigm and exposure with base-out prisms to evaluate the relative contributions of sensory (visual and proprioceptive) and motor components of adaptation to perceived-distance alteration. A main experiment was designed to elicit adaptation at the sensory and motor levels, by giving subjects altered visual feedback. A control experiment without visual feedback allowed the effects of eye muscle potentiation (EMP) induced by sustained fixation through the prisms to be uncovered. In the main experiment, the aftereffects were partitioned into two-thirds visual and one-third motor, with no significant proprioceptive component. These results differ from the classical pattern of short-term adaptation to prism-altered apparent visual direction, which includes mainly proprioceptive/motor adaptive components, with a smaller visual component. This difference can be attributed to differences in accuracy between proprioception and vision for localization in depth or in lateral directions. In addition, a comparison of the visual aftereffects in the main and control experiments revealed two sub-components with equal contributions: a recalibration of the mapping between the vergence signal and perceived distance, and an EMP-related aftereffect. These findings reveal that "visual" adaptation actually involves a multiplicity of processes.
\end{abstract}

Keywords: adaptation; prism; reaching; distance perception; vergence; cue weight 


\section{Introduction}

Reaching forward to grasp an object or to point to a target requires an accurate estimation of their distance and direction. During development and throughout life, or after peripheral or central lesions, perception and action adapt to take into account changing optical and physiological signals, which convey information about the direction and distance of stimuli.

When viewing their moving hand through wedge prisms that displace the visual field laterally, subjects correct initial errors in reaching after a few tens of trials. When the prisms are removed and subjects are asked to reach for an object, they usually miss the target, with an error in the direction opposite to that of the displacement previously induced by the prism. This compensatory change in eye-hand coordination is called the 'visuomotor negative aftereffect' (see Kornheiser, 1976; Welch, 1986 for reviews). The classical prism paradigm pioneered by Helmholtz (1910) involves pre-exposure (baseline) measurement of visuomotor performance, exposure to prismatic displacement, and then post-exposure measurement. The visuomotor aftereffect is defined as the signed difference between pre- and post-exposure measures, and reflects visuomotor adaptation.

Visuomotor adaptation to prism-induced alteration of apparent visual direction involves various adaptive components (see Baraduc \& Wolpert, 2002; Welch, 1986 for reviews). The first component, proprioceptive adaptation, is a change in the felt position of the limb relative to the body (Harris, 1963, 1965). The second component is motor adaptation (Baily, 1972; Morton \& Bastian, 2004; Templeton, Howard, \& Wilkinson, 1974; Wallace \& Redding, 1979). It implies a reorganization of the muscle commands associated with a given visual goal (Baraduc \& Wolpert, 2002; Kitazawa, Kimura, \& Uka, 1997; Martin, Keating, Goodkin, Bastian, \& Thach, 1996b). The third component, visual adaptation, is a change in apparent visual direction (Welch, Choe, \& Heinrich, 1974; Wilkinson, 1971). It involves a recalibration of the felt direction of gaze (Craske, 1967; Helmholtz, 1910; Kalil \& Freedman, 1966) and of the head-on-shoulder position (Hamilton, 1964; Harris, 1965; Kornheiser, 1976).

Proprioceptive adaptation occurs in the early stage of prism exposure (Harris, 1963; Hay \& Pick, 1966), whereas visual adaptation requires a longer time to develop (Hay \& Pick, 1966). Motor adaptation, generally observed with fast movements, develops in the very-short term (Day \& Singer, 1967; Martin, et al., 1996b). Thus, all or most of the adaptive short-term changes in eye-hand coordination following prism-altered apparent visual direction have been attributed to proprioceptive and motor adaptation, with little or no visual adaptation. Note that in the studies cited above, the proprioceptive component usually included both motor recalibration and proprioceptive adaptation effects.

The mechanisms of visuomotor adaptation to prism-altered apparent visual direction have been widely investigated (see Redding \& Wallace, 2006; Welch, 1986 for reviews). By contrast, little is known about the mechanisms underlying prism-induced alteration of perceived distance. While a lateral deviation of visual direction is produced by placing wedge prisms with the same orientation in front of both eyes (hereafter referred to as lateral prisms), opposite-base wedge prisms modify egocentric distance perception by manipulating the vergence required to fuse objects. The main purpose of the present study was to investigate visuomotor adaptation to prism-altered perceived distance and to isolate its components. A secondary goal was to compare these components with those classically described for prismaltered apparent visual direction under similar experimental conditions. 
To partition the components of visuomotor adaptation to prism-altered perceived distance, two experiments were performed. The main experiment was designed to elicit adaptive components at various sensory and motor levels, by giving subjects altered visual feedback. During the exposure period, subjects pointed to visual targets while viewing through a pair of base-out prisms. Base-out prisms decrease vergence-specified egocentric distances (see Fig. 1a). This type of exposure initially causes a discrepancy between felt and seen hand position: the hand is seen as closer than indicated by proprioceptive information. This intersensory discrepancy induces an adaptive process to minimize the conflict between visual and proprioceptive information. This adaptive process should lead to a progressive increase in visually perceived distance and/or a foreshortening of proprioceptive distance. After removal of the prisms, a visual shift towards greater perceived distance (hereafter referred to as visual recalibration) and/or a proprioceptive shift towards a nearer position (hereafter referred to as proprioceptive adaptation) are therefore expected (see Fig. 1b). In addition, an inconsistency between the visual goal and the actual movement endpoint may drive a motor adaptation process, even without inter-sensory conflict (Magescas \& Prablanc, 2006). Specifically, subjects are expected to under-reach the target at the beginning of exposure (see Fig. 1a). Thus, the adaptive response should consist of a progressive decrease in the under-reach error during exposure. After removal of the prisms, an over-reach motor response (hereafter referred to as motor adaptation) is expected (see Fig. 1b). In the current study, visual, proprioceptive and motor components of adaptation to prism-altered perceived distance were isolated through a series of coordination tests (Harris, 1965). In accordance with the additivity hypothesis, the visuomotor aftereffect was considered to be the sum of the visual, proprioceptive and motor components (see Redding \& Wallace, 1992; Welch, 1974; 1986 for reviews).

Another source of changes in perceived distance, which is unrelated to inter-sensory conflict, is eye muscle potentiation (EMP). Sustained convergence through the prisms is sufficient to induce changes in extraocular muscles tonus (i.e., increased tonic vergence) and a subsequent increase in perceived distance (Ebenholtz, 1981; Owens \& Leibowitz, 1980;

Paap \& Ebenholtz, 1977). To investigate the contribution of EMP to the visual aftereffects under study, a control experiment was performed. In this experiment, in contrast to the main experiment, visual feedback of the hand was not available during the exposure period. EMPrelated aftereffects in the two experiments should be similar, as the target sequence and visual and oculomotor tasks were exactly the same. Thus, by comparing visual aftereffects in the main and control experiments, it was possible to assess the visual recalibration component (Priot, Laboissière, Sillan, Roumes, \& Prablanc, 2010).

\section{Materials and methods}

\subsection{Subjects}

Thirteen subjects, including six females and seven males (ages ranging from 21 to 45 years, with a mean of 28 years), took part in the main experiment. All subjects except one were right-handed. All were screened for stereoscopic vision (TNO $\leq 60$ '), and none of them had a history of binocular disorders. All had normal or corrected-to-normal vision. Prescribed correction, if any, was worn during the experiments. All subjects provided informed consent prior to participation. The experiments were conducted in accordance with the Declaration of Helsinki and under the terms of local legislation. 


\subsection{Apparatus}

During exposure, subjects wore a pair of 5- $\Delta$ base-out wedge prisms mounted on goggles. This optical arrangement displaces the lines of sight of each eye inwards, thereby increasing binocular convergence by $5.71^{\circ}$ (Fig. 1a). This $5-\Delta$ deviation was chosen because it is the value most commonly used in the literature (Ebenholtz, 1981; von Hofsten, 1979; Wallach \& Frey, 1972; Wallach, Frey, \& Bode, 1972). An immediate side-effect of the prisms was a reduction of the apparent size of objects (Howard \& Rogers, 2002). The stimulus to accommodation was not altered by the prisms. Although adaptation to perceived-distance alteration induced by base-in prisms is also of interest, we decided against the use of base-in prisms in the current study because one of the goals of this study was to compare adaptation to alterations of apparent distance and visual direction under similar experimental conditions. Base-in prisms would change the range of registered vergence distances from $353-500 \mathrm{~mm}$ to $789-2272 \mathrm{~mm}$. The latter includes distances that are outside of the range of hand pointing, and would have required a different test paradigm. We chose a hand-pointing paradigm so that our results would be comparable to those obtained in previous studies using lateral prisms, which have also used hand-pointing paradigms (see Welch, 1986 for review).

Figure 2 shows the experimental setup. The visual stimulus was produced using a red lightemitting diode (LED) placed on a ramp located vertically above the subject's head. As the subject observed the target through a central half-silvered mirror tilted $45^{\circ}$ with respect to the vertical fronto-parallel plane, the vertical ramp of the target appeared horizontal. There was neither finger-to-target masking nor any tactile feedback, as the target was a virtual image. Direct vision through the mirror could be prevented by an occluding screen placed behind the mirror. A mask with cross-hairs slits was placed in front of the target, which appeared as a luminous red cross. A diaphragm, disposed just in front of the target, could reduce the diameter of the cross to a 1-mm red luminous point. In both cases, the stimulus to accommodation was poor. The target could move on a motorized ramp along a horizontal axis in the sagittal plane at the ocular level. Head movements were restrained using forehead and chin rests.

Pointing movements were unconstrained. A 2-mm infrared-emitting diode (900-nm wavelength) was attached to both index fingertips, which $3 \mathrm{D}$ positions were recorded at $250 \mathrm{~Hz}$ with an Optotrak 3020, Northern Digital Inc. The apparatus was calibrated using the Optotrak. A LED marker was mechanically displaced until two experimenters on both right and left sides of the half-silvered mirror judged it to be coincident with the target image seen through the mirror. The accuracy of this procedure was smaller than $1 \mathrm{~mm}$.

\subsection{Procedure}

A classical paradigm including three blocked conditions of pre-test, active exposure to baseout prisms and post-test was carried out in an otherwise dark room. The exposure involved closed-loop pointing with the left hand only; the right hand was out of view.

\subsubsection{Pre- and post-tests}

The tests for eye-hand coordination were performed during the pre- and post-tests of the main experiment. Prior to the experiment proper, subjects were given the opportunity to familiarize 
themselves with the pointing task, and they were trained to point to a visual target with each hand successively, first with visual feedback of their hand, then without such feedback.

Visuomotor aftereffect was assessed by measuring pre-to-post changes in open-loop pointing (i.e., pointing without visual feedback of the hand) to visual targets with the left (exposed) hand (Harris, 1965). Visual aftereffect was assessed by measuring pre-to-post changes in open-loop pointing to visual targets with the right (unexposed) hand (Harris, 1965; Priot, et al., 2010). The use of the right (unexposed) hand aftereffect to isolate the visual component of adaptation is supported by earlier findings showing complete visual transfer from the exposed to the unexposed hand (Kornheiser, 1976; McLaughlin \& Bower, 1965; Wallace \& Redding, 1979) and no proprioceptive or motor transfer (Hamilton, 1964; Harris, 1963, 1965; Kitazawa, et al., 1997; Martin, et al., 1996b; Prablanc, Tzavaras, \& Jeannerod, 1975). Therefore, using the unexposed right hand for pointing underscores the complete visual component of adaptation, uncontaminated by short-term visuomotor adaptation of the left exposed hand. Subjects were instructed to avoid all contact between their left and right hands during the experiment in order to prevent any interlimb proprioceptive and/or motor transfer. For these tests, a red punctual target was displaced 270 to $495 \mathrm{~mm}$ from the subject's cornea in 5-mm increments, in a pre-determined random order common to all subjects. Each target location was presented once, resulting in 46 trials. A single target was lit at a time. A 2-s dark interval between the offset of a target and the onset of the next one was intended to reduce disparities between successive stimuli. A beep cued the beginning of each trial. Subjects were instructed to point their left-index fingertip (in the visuomotor test) or their right-index fingertip (in the visual test) as accurately as possible to the perceived location of the target, starting from a rest position close to the chest. They validated their response by pushing a button with their stationary hand and returned their pointing hand to the rest position. Each test lasted about four minutes.

Proprioceptive aftereffect on the left hand was assessed by measuring pre-to-post changes in pointing to the left (exposed) fingertip with the right (unexposed) fingertip while keeping the eyes closed (Craske, 1966; Cressman \& Henriques, 2009; Harris, 1965; Simani, McGuire, $\&$ Sabes, 2007; Welch, 1969). This method makes it possible to assess proprioceptive aftereffect without any motor contamination. This might not be the case for straight-ahead pointing, which has been suggested to involve recalibration of motor outflow, in addition to the passively measurable proprioceptive component (Hatada, Miall, \& Rossetti, 2006; Lester, 1968). The subject's left-index fingertip was placed by the experimenter at the mid-distance of the testing range ( $380 \mathrm{~mm}$ from the subject's cornea). Subjects were instructed to point as accurately as possible with their right-index fingertip to the felt location of their left-index fingertip, keeping a frontal gap of a few $\mathrm{cm}$ between the two indexes to avoid contact. They validated their response by pushing a button with their left thumb and returned the right hand to the rest position. An interval of 3-s separated the response validation from the onset of the next trial, which was cued by a beep. Ten trials started with the rest position close to the chest (near-to-far pointing). For the following 10 trials, the starting position involved extending the arm (far-to-near pointing). The positions of the two indexes were recorded.

Motor aftereffect was estimated indirectly, by subtracting the visual and proprioceptive aftereffects from the visuomotor aftereffect, consistent with the additivity hypothesis. Similar methods have been used to estimate the proprioceptive (Baily, 1972) and visual aftereffects (McLaughlin \& Bower, 1965; McLaughlin, Rifkin, \& Webster, 1966). 
Before and after eye-hand coordination tests, far phoria, as a measure of tonic vergence (Judge \& Bradford, 1988; Judge \& Miles, 1985), was assessed using the Maddox-rod technique. Pre-to-post changes in phoria quantified the effects of EMP (Ebenholtz, 1981; Shebilske, Karmiohl, \& Proffitt, 1983).

During pre-test, phoria measurements were performed first, followed by the proprioceptive test, followed by the visuomotor and visual tests, the order of the latter two tests being counterbalanced across subjects. During post-test, the visuomotor and visual tests were performed first (their order being counterbalanced across subjects), followed by proprioceptive test, and finally the phoria test.

\subsubsection{Prism-viewing exposure}

The left hand was used for pointing during prism-viewing exposure. Visual feedback was allowed by removing the occluding screen behind the half-silvered mirror. Instead of directly seeing their left fingertip, subjects saw a green LED attached to their left-index fingertip in an otherwise dark room. This was intended to prevent the use of familiar hand- or finger-size cues, which could be altered by the prisms. Vergence (associated with horizontal disparity) was the only available visual cue to distance. Vertical disparities are unlikely to have played a role since targets were located along a horizontal axis in the sagittal plane at the ocular level and the fingertip LED was not seen during the major part of the movement, due to the restricted field of view through the apparatus. The stimulus was a red luminous cross (1-mm width, 3-mm height). This stimulus shape was selected to favor fusion, and to provide a stimulus different from that used during the pre- and post-tests, thus preventing size cues. The target was displaced 353 to $500 \mathrm{~mm}$ from the subject's cornea in 3-mm increments following a pre-determined random order, which was common to all subjects. Each target location was presented twice, resulting in 100 trials. Starting from a rest position close to the chest, subjects were instructed to point their left-index fingertip at the perceived location of the target and to adjust their movement until the fingertip LED visually coincided with the cross target. They validated their response by pushing a button with their right thumb and brought their left hand back to the rest position. The prism-viewing exposure phase lasted about ten minutes.

\subsection{Control experiment}

Inclusion criteria and procedure for the control experiment were identical to those used in the main experiment, with the following exceptions. Fourteen right-handed subjects, including 10 females and 4 males (ages ranging from 18 to 34 years, with a mean of 24 years) took part in the control experiment. Five subjects participated in both the main experiment and the control experiment, with a delay of at least two months between the experiments. During exposure to base-out prisms, visual feedback of the left hand was not available while subjects were pointing to visual targets. Because the aim of this control experiment was to assess visual (EMP-related) aftereffect, pre- and post-tests involved only open-loop pointing to visual targets with the right (unexposed) hand.

\subsection{Data analysis}

For each trial, the fingertip position was averaged over the 40-ms interval following the onset of the validation-button push. Egocentric distance was measured as the distance along the Y- 
axis (horizontal) in the sagittal plane located at eye level (Mon-Williams \& Tresilian, 1999), the origin of which was in the coronal plane tangential to the subject's cornea.

The functional relationship between actual and estimated target distances was analyzed using a linear model (Ebenholtz, 1981; Mon-Williams \& Tresilian, 1999). For each subject and each condition, a linear model using a least-squares criterion was applied between the distance estimated by pointing (dependent variable) and target distance (independent variable). The mean pointing distance was defined as the value predicted by the linear fit at the mean target distance $(382.5 \mathrm{~mm})$. For pointing responses to visual targets, the aftereffect was defined as the signed difference between pre- and post-test mean pointing distances. Left- and right-hand pointing aftereffects represented the visuomotor and visual aftereffects, respectively. For each trial of the proprioceptive test in the main experiment, we computed the difference between the right and left fingertip positions along the $\mathrm{Y}$ axis, which was the relative distance between both fingertips. Mean relative distances for both pre- and post-tests were then computed. The proprioceptive aftereffect was defined as the signed difference between these two mean distances. For each subject in the main experiment, the motor aftereffect was calculated by subtracting the visual and proprioceptive aftereffects from the visuomotor aftereffect. The visual recalibration component was obtained by subtracting the visual aftereffect in the control experiment from the visual aftereffect in the main experiment (Priot, et al., 2010).

Any change in the adaptive direction was scored positive (see Fig. 1b). Our hypotheses related to the sign of the aftereffect justified the use of one-sided t-tests. The level of statistical significance was set at 0.05 .

\section{Results}

\subsection{Main experiment}

\subsubsection{Visuomotor aftereffect (left-hand pointing to a visual target) and visual aftereffect (right-hand pointing to a visual target)}

In Fig. 3, distance estimation is plotted as a function of target distance for left- and right-hand pointing and for pre- and post-tests. The mean value for the exposed left-hand pointing aftereffect was $65.6 \mathrm{~mm}(\mathrm{SE}=10.7 \mathrm{~mm})$ and was significantly larger than zero $(\mathrm{t}[12]=6.12$, $\mathrm{p}<0.001$, one-sided paired t-test). This was also the case for the mean value of $47.9 \mathrm{~mm}$ $(\mathrm{SE}=6.7 \mathrm{~mm})$ for the unexposed right-hand pointing aftereffect $(\mathrm{t}[12]=7.11, \mathrm{p}<0.001)$.

\subsubsection{Proprioceptive aftereffect (right-hand pointing to left hand)}

The mean value of $-1.2 \mathrm{~mm}(\mathrm{SE}=2.4 \mathrm{~mm})$ for the proprioceptive aftereffect was not significantly larger than zero $(\mathrm{t}[12]=-0.496, \mathrm{p}>0.3$, one-sided paired $\mathrm{t}$-test).

\subsubsection{Calculated motor aftereffect}

The sum of the visual and proprioceptive aftereffects was significantly smaller than the visuomotor aftereffect $(\mathrm{t}[12]=3.1, \mathrm{p}<0.01$, one-sided paired $\mathrm{t}$-test $)$, which supports the existence of a motor component in the expected direction. The mean value for the computed motor aftereffect was $18.9 \mathrm{~mm}(\mathrm{SE}=6.1 \mathrm{~mm})$. 


\subsection{Control experiment}

Distance estimation as a function of target distance in the control experiment is represented in Fig. 4. The mean value for the aftereffect in the control experiment was $27.3 \mathrm{~mm}$ $(\mathrm{SE}=5.9 \mathrm{~mm})$ and was significantly larger than zero $(\mathrm{t}[13]=4.64, \mathrm{p}<0.001$, one-sided paired t-test).

\subsection{Comparison between the main and control experiments' visual aftereffects}

The difference between the visual aftereffects in the main and control experiments was assessed using a non-paired one-sided t-test. The aftereffect measured in the main experiment was $20.6 \mathrm{~mm}$ larger than the aftereffect measured in the control experiment, and this difference was significantly larger than zero $(\mathrm{t}[25]=2.3, \mathrm{p}<0.02)$. The mean change in phoria during the control experiment was $1.6 \Delta(\mathrm{SE}=0.2 \Delta)$, which was also significantly larger than zero $(t[13]=6.9, p<0.001$, one-sided paired $t$-test $)$. This indicates a change towards esophoria (i.e., a more convergent eye position). The mean change in phoria measured in the main experiment, $1.6 \Delta(\mathrm{SE}=0.4 \Delta)$, was also statistically significant $(\mathrm{t}[12]=3.9, \mathrm{p}<0.002)$. Equal mean changes in phoria in the main and control experiments indicate an equal contribution of EMP to the visual aftereffects in both experiments. The 20.6-mm difference between the aftereffects measured in these two experiments corresponds to the visual recalibration component. Figure 5 summarizes the partitioning of the components of visuomotor adaptation.

\section{Discussion}

The main finding of the present study is a particular pattern of visuomotor adaptation following exposure to prism-altered perceived distance. The results indicate that approximately one-third (29\%) of the aftereffect measured in the main experiment reflected the contribution of a motor component. The remaining $71 \%$ (approximately two-thirds) of the aftereffect reflected a visual component. No significant contribution of a proprioceptive component was found. This pattern differs from the classical pattern of short-term visuomotor adaptation to prism-altered apparent visual direction, the main component of which is proprioceptive/motor, with little or no contribution from visual adaptation.

\subsection{The nature of the visual component}

It has been proposed that visual adaptation to prism-altered apparent visual direction results from changes in felt direction of gaze, which can be divided into two sub-components: eye muscle potentiation (EMP) (Ebenholtz, 1974), and a recalibration of visual direction (Craske \& Crawshaw, 1975). A prolonged deviation of the eyes from their normal rest position tends to reflexively maintain this ocular position even after relaxing the eyes, a phenomenon referred to as eye muscle potentiation. The efference required to bring back the eyes from their EMP-induced deviation to straight-ahead induces a misperception of the felt direction of gaze in the opposite direction (Ebenholtz \& Wolfson, 1975). The recalibration of visual direction involves a remapping between eye deviation and the felt direction of gaze elicited by cue-discrepancy experience (Craske \& Crawshaw, 1975, 1978). Whether the felt direction of gaze derives from an oculomotor efferent copy and/or an extraocular proprioceptive signal is still a matter of debate. There is however some evidence that both efferent and proprioceptive 
information combine to code eye position information during slow eye movements (see Donaldson, 2000 for review; Gauthier, Nommay, \& Vercher, 1990a, 1990b; Steinbach $\&$ Smith, 1981). A change in retinal local sign could also potentially lead to a change in apparent visual direction (see Welch, 1986 for review). However, results about this hypothesis are both sparse and conflicting (Cohen, 1966; Craske \& Crawshaw, 1974; Howard, 1970). Even though visual adaptation following exposure to prism-altered apparent visual direction appears to be a type of altered eye position sense, it can be considered as a change in vision because it has the same phenomenal consequences for visual perception (Welch, 1986).

Similarly to the visual component of adaptation to prism-altered apparent visual direction, the visual component of adaptation to prism-altered perceived distance studied here may be partitioned into two sub-components. The first is a distance estimation aftereffect resulting from an altered vergence effort following sustained convergence (Priot, et al., 2010). This sub-component, which may be attributed to a change in extraocular muscles tonus (Ebenholtz, 1974, 1981; Ebenholtz \& Wolfson, 1975; Paap \& Ebenholtz, 1977), was obtained in our control experiment without any feedback during exposure. Sustained convergence below the physiological position of rest (PPR), which corresponds to a distance of $30 \mathrm{~cm}$, is known to increase tonic vergence (Ebenholtz, 1974; Ebenholtz \& Wolfson, 1975; Paap \& Ebenholtz, 1977). When trying to keep fixation onto a target, a greater divergence effort is necessary to overcome the increased tonic vergence. This change in efference results in distance overestimation, as revealed by the EMP-related aftereffect in our control experiment (Ebenholtz, 1981; Ebenholtz \& Fisher, 1982; Owens \& Leibowitz, 1980; Shebilske, et al., 1983). The second visual sub-component is an active visual-adaptation process induced by cue-discrepancy experience (Craske \& Crawshaw, 1974; Wallach, et al., 1972; Wallach \& Smith, 1972). In contrast with the control experiment, in the main experiment, visual feedback of the hand was available during exposure. The conflict between altered vergence/disparity and unaltered limb proprioception/motor cues gave rise to a recalibration of the mapping between the vergence signal and egocentric perceived distance (herein referred to as vergence/distance mapping). A recalibration of the mapping between disparity and relative perceived distance is unlikely to have occurred. Indeed, aftereffects in perceived relative distance have been shown to derive from changes in vergence/distance mapping (Fisher \& Ciuffreda, 1990; Fisher \& Ebenholtz, 1986). As the vergence signal for distance depends upon either extraocular muscle proprioception and/or oculomotor efference copy (Collewijn \& Erkelens, 1990; Owens \& Leibowitz, 1980; von Hofsten, 1976), the recalibration of the vergence/distance mapping may have occurred at these two stages.

The visual aftereffect observed in the current study can account for approximately two thirds of the visuomotor aftereffect. This is consistent with earlier findings (Wallach \& Smith, 1972). Our results suggest that an interpretation of the visual aftereffect in terms of visual recalibration induced by cue-discrepancy experience is incomplete (see also Epstein, 1975). A significant component of the visual aftereffect appears to be related to neuromuscular mechanisms. The contributions of EMP to the visual and visuomotor aftereffects are far from being negligible: they amount to 55\% and 30\%, respectively. EMP is a rapid and efficient mechanism that reduces the impact of prism-altered perceived distance, thereby reducing the need for visual and visuomotor recalibration within reaching space.

\subsection{Sources of inconsistency subserving the different components of adaptation}

Different sources of inconsistency during exposure, including spatial inconsistency and 
reaching errors, could have contributed to the different components of adaptation. The sources of inconsistency were: (1) a discrepancy between vision and fingertip proprioception (Craske \& Crawshaw, 1974; Redding \& Wallace, 1992); (2) an inconsistency between actual (visual) and expected (derived from the efferent copy) feedback of the fingertip, as suggested by Held's efference-reafference theory (1958); (3) a terminal reaching error given by the relative fingertip-to-target disparity (Kitazawa, Kohno, \& Uka, 1995; Magescas \& Prablanc, 2006). Prism-altered apparent visual direction also involves the three sources of errors mentioned above.

In our main experiment, the recalibration of the vergence/distance mapping likely arose from inter-sensory inconsistencies (Wallach \& Smith, 1972). The discrepancy between felt and seen limb position (item 1) may represent a primary source of adaptation for both apparent distance and direction alterations. Visual-proprioceptive discrepancy arises from the spatial distortion caused by the prisms, which results in translation or compression of the visual world. To a first approximation, lateral prisms produce only a translation alteration in the fronto-parallel plane. By contrasts, opposite-base prisms produce a compression alteration. Compression alteration introduces velocity discrepancies, which are absent in translation alteration. Accordingly, a larger discrepancy in visual-proprioceptive information may induce a stronger visual-proprioceptive recalibration for distance alteration than for direction alteration. However, amplitude and velocity components likely played a minor role in the present experiment, as the hand was seen only in the deceleration phase of the movement.

The relative contributions of sensory adaptive components to prism-induced alterations of either distance or direction may be explained in terms of the relative accuracy of vision and proprioception. The accuracy of vergence sensing has been reported to be comparable to the accuracy of versional eye-position sensing (see Collewijn \& Erkelens, 1990 for review). However, as predicted based on geometrical considerations, similar angular accuracies in vergence and version result in poorer distance estimation than direction estimation, when expressed in Cartesian coordinates (Brenner \& Smeets, 2000). Moreover, when subjects fuse information from two different sensory modalities (for hand or target localization), the weights assigned to each signal are proportional to the accuracy in the respective modality, as has been shown in visual-auditory (Ghahramani, Wolpert, \& Jordan, 1997), visual-haptic (Burge, Girshick, \& Banks, 2010) and visual-proprioceptive (van Beers, Wolpert, \& Haggard, 2002) adaptation experiments. In addition, the results of these experiments have shown that adaptation aftereffects are larger in the less accurate modality. Based on these results, one could expect a larger visual adaptation component in our main experiment than in adaptation experiments using lateral prisms. This is consistent with previous studies showing that visual information is less important for the control of movement distance than for the control of movement direction (Bagesteiro, Sarlegna, \& Sainburg, 2006; Sarlegna, et al., 2003). Moreover, the lack of a proprioceptive aftereffect in our main experiment may be explained by a stronger weight of limb proprioception compared to vision, when these modalities are used for localization in depth. This is consistent with the results of van Beers et al. (2002) in an adaptation paradigm to computer-alteration of apparent distance and direction: the visual component of adaptation was over twofold larger than the proprioceptive component for distance alteration, whereas the reverse pattern was observed for direction alteration.

It is worth noting that regardless of the studied dimension (i.e., lateral vs in-depth), reducing visual cues has been found to result in smaller weights assigned to visual information, while adding visual cues has been found to increase reliance on visual information (Mon-Williams, Wann, Jenkinson, \& Rushton, 1997; Plooy, Tresilian, Mon-Williams, \& Wann, 1998). 
Similarly, the type of visual feedback (concurrent vs terminal) during exposure also affects the nature of adaptive components to lateral prisms. Early visual feedback (concurrent exposure) during movement favors proprioceptive adaptation while delayed visual feedback (terminal exposure) at the end of movement favors visual adaptation (see Redding, Rossetti, \& Wallace, 2005 for review). During concurrent exposure, the early and continuous availability of visual information likely increases the weight of vision. Conversely, visual feedback was almost restricted to the end of movement in our main experiment. These remarks concerning the amount of visual information and the type of visual feedback are consistent with reliability-based cue weighting models (see Ernst \& Bülthoff, 2004 for review; Landy, Maloney, Johnston, \& Young, 1995; Mamassian, Landy, \& Maloney, 2002).

The last component of adaptation, motor recalibration, develops quickly from the efferencereafference conflict (item 2 above; Held \& Hein, 1958) and/or from the terminal reaching error (item 3; see Kornheiser, 1976 for review). The salience of the efference-reafference conflict (item 2) is potentially lower for direction alteration than for distance alteration (Howard, 1970, 1971). Indeed, the motor outflow determines the amplitude, velocity and direction of arm movement. The two former aspects are not affected by lateral prisms. However, as for visual-proprioceptive discrepancy (item 1), the roles of amplitude and velocity components were limited to the deceleration phase of the movement. In addition, direction and distance alterations yield qualitatively different terminal reaching errors (item 3). In direction alteration, the pointing error is immediately specified by terminal fingertip-to-target errors on each retina. In distance alteration, the pointing error has to be computed from the fingertip-to-target disparity (Snijders, Holmes, \& Spence, 2007). For a given amount of pointing error (in Cartesian coordinates), the (angular) retinal error is smaller for distance alteration than for direction alteration. This difference might lead to a smaller relative contribution of the motor component in our main experiment as compared to prismaltered apparent direction.

The different patterns of adaptation for distance and direction alterations may be related to neurophysiological findings in monkeys, and neuroimaging and clinical findings in humans. Recent fMRI (Quinlan \& Culham, 2007) and clinical studies (Coello, Danckert, Blangero, \& Rossetti, 2007; Danckert, Goldberg, \& Broderick, 2009) have suggested that the control of movement in the sagittal plane differs from that of movement in the fronto-parallel plane. In a recent review, Ferraina et al. (2009) emphasized the role of the parietal cortex for coding action in depth. Bhattacharyya et al. (2009) have suggested that the parietal region encodes reach distance through a modulation of retinal disparity by vergence at the neuronal population level. Various subregions of the parietal cortex control distinct components of visuomotor behavior in the sagittal plane (see Ferraina, et al., 2009 for review). Quinlam \& Culham (2007) have shown preferential coding of moving stimuli in near versus far space in the dorsal region of the parieto-occipital sulcus (dPOS), the near response being probably determined in large part by vergence. In addition, differential coding of movements towards and away from the body appears to involve area V6A of the monkey parietal cortex (Galletti, Fattori, Gamberini, \& Kutz, 1999). Similarly, Danckert et al. (2009) reported that human movements in the sagittal plane are more impaired following right superior parietal damage than movements in the fronto-parallel plane. While the neural processes underlying adaptation to prism-altered apparent visual direction have been widely investigated (Baizer, Kralj-Hans, \& Glickstein, 1999; Luauté, et al., 2009; Martin, Keating, Goodkin, Bastian, \& Thach, 1996a; Prevosto, Graf, \& Ugolini, 2009; Weiner, Hallett, \& Funkenstein, 1983), little is known about the neural processes underlying adaptation to prism-altered perceived distance. To our knowledge, the only data currently available on this issue relate to the EMP-related 
aftereffect. Morley et al. (1992) have suggested that the increase in tonic vergence observed after exposure to sustained vergence is mainly mediated by vergence-related neurons (nearresponse neurons, see Cumming \& Judge, 1986) in the midbrain.

\section{Conclusion}

The present study showed a three-level adaptation to prism-altered perceived distance: 1) an EMP-related aftereffect in response to sustained increased vergence; 2) a recalibration of the mapping between the vergence signal and perceived distance; and 3) a motor recalibration. The first two levels are related to changes in visual perception common to both hand effectors, whereas the third is related to changes in the motor commands of the exposed limb only.

Contrary to the classical assumption that vision dominates the other senses, vision appears to be quite flexible under short-term exposure to inter-sensory discrepancy, while proprioception remains unchanged. Visual recalibration occurred in response to altered visual feedback, the weight of vision being lower than the weight of limb proprioception for localization in depth. EMP contributed to half of the observed visual aftereffect and to one-third of the observed visuomotor aftereffect. This component, which arises independently of any inter-sensory or sensorimotor discrepancies, should be appropriately quantified in adaptation studies involving unusual sustained eye positions.

\section{Acknowledgements}

This work was supported by grant $\mathrm{N}^{\circ} 07 \mathrm{CO} 802$ from the Délégation Générale pour l'Armement. The experiments described in this article were performed in the "Plateforme Mouvement et Handicap" HCL-IFNL (Lyon, France). The authors would like to thank Olivier Sillan for software and electronics development, Véronique Chastres for help with the statistical analysis, and James Danckert and an anonymous reviewer for helpful suggestions.

\section{Legends}

Fig. 1. Prism-induced alteration of perceived distance. (a) Course of the prism exposure period from $t_{\mathrm{o}}$ to $t_{\text {end }}$. The subject looks at a visual target $\mathrm{A}$, located along a sagittal line. Fixation at $\mathrm{A}$ through the prisms requires the convergence of the two lines of sight onto B, causing A to appear at B. At the first attempt for the left hand to point toward A (seen at B), the subject reaches for $\mathrm{B}$ and sees his/her hand at $\mathrm{C}$, closer to his/her eyes (green hand: physical position of the hand; rose hand: seen position of the hand). After tens of iterative movements, the subject finally reaches for A, while both target and hand are seen at B. (b) Comparison between pre- and post-tests. After prisms removal, vision of either hand is occluded and the subject undergoes several tests. The proprioceptive test consists of pointing with the right (unexposed) hand (green hand with an arrow) to the left (exposed) hand (green hand). When required to point in the dark with the right hand toward the left one located in the mid-sagittal plane, the subject points to a closer location, with an inward shift represented by a vector $P$. Then, a visual test consists of pointing with the unseen right hand to a visual target located at D. An adaptive response corresponds to an outward deviation towards $\mathrm{E}$ with a vector $\mathrm{V}$. The last visuomotor test consists of pointing to visual target $\mathrm{D}$ with the unseen left hand. The adapted subject points outwards to F, with a vector VM. Even in the case of a fully 
adapted subject, VM is always smaller than the prism alteration delta due to the disappearance of the cognitive effect after prisms removal. The motor component $\mathrm{M}$ is inferred from the previous measures and is equal to $|\mathrm{VM}|-(|\mathrm{P}|+|\mathrm{V}|)$.

Fig. 2. Experimental setup. The subject is looking through the prisms while resting his/her head and chin. The pronated right (or left) hand points to the estimated position of the target behind the half-silvered mirror. The physical image of the LED target is shown as a red solid cross at A, in correspondence to the LED target along the vertical ramp. The vergencespecified location of the target seen through the prisms is represented as a red dashed cross at B. During pre- and post-tests, the subject does not see his/her hand due to the occluding screen placed behind the mirror. During exposure, the occluding screen is removed and the subject can see a green LED placed on his/her left fingertip (not shown).

Fig. 3. Distance estimation as a function of target distance in the main experiment. Each point represents the mean value of the pointing distance (vertical axis) for each target position (horizontal axis), in each condition (red filled circles: left hand pre-test; blue open circles: right hand pre-test; red filled triangles: left hand post-test; blue open triangles: right hand post-test). Standard errors are not indicated for clarity. Regression lines are shown for each condition.

Fig. 4. Distance estimation as a function of target distance in the control experiment. Each point represents the mean value of the pointing distance (vertical axis) for each target position (horizontal axis), in each condition (blue open circles: right hand pre-test; blue open triangles: right hand post-test). Standard errors are not indicated for clarity. Regression lines are shown for each condition.

Fig. 5. Components of visuomotor adaptation to prism-altered perceived distance. On the left, measured components appear as vertical bars. The visuomotor, visual and proprioceptive aftereffects were obtained in the main experiment, while the EMP-related aftereffect was obtained in the control experiment. Standard errors are indicated by vertical bars. Positive values indicate changes in the adaptive direction. Asterisks indicate a significant aftereffect. On the right, partitioning of components is represented as vectors. Positive values reveal changes in the adaptive direction. The left red solid arrow represents total visuomotor aftereffect labeled VM. The middle arrows represent the components of visuomotor aftereffect: the visual aftereffect labeled V (green long dashed arrow), the motor aftereffect labeled M (blue short dashed arrow) and the non-significant proprioceptive aftereffect labeled $\mathrm{P}$ (brown arrow). The motor aftereffect exceeds the difference between the visuomotor and visual aftereffects as it is calculated by the subtraction of the visual and proprioceptive aftereffects from the visuomotor aftereffect. The right arrows represent the sub-components of the visual aftereffect: the recalibration component labeled Recal. (green dashed and dotted arrow) and the EMP-related aftereffect labeled EMP (green dotted arrow). 


\section{References}

Bagesteiro, L. B., Sarlegna, F. R., \& Sainburg, R. L. (2006). Differential influence of vision and proprioception on control of movement distance. Experimental Brain Research, 171, 358-370.

Baily, J. S. (1972). Adaptation to prisms: do proprioceptive changes mediate adapted behaviour with ballistic arm movements? The Quarterly Journal of Experimental Psychology, 24, 8-20.

Baizer, J. S., Kralj-Hans, I., \& Glickstein, M. (1999). Cerebellar lesions and prism adaptation in macaque monkeys. Journal of Neurophysiology, 81, 1960-1965.

Baraduc, P., \& Wolpert, D. M. (2002). Adaptation to a visuomotor shift depends on the starting posture. Journal of Neurophysiology, 88, 973-981.

Bhattacharyya, R., Musallam, S., \& Andersen, R. A. (2009). Parietal reach region encodes reach depth using retinal disparity and vergence angle signals. Journal of Neurophysiology, 102, 805-816.

Brenner, E., \& Smeets, J. B. (2000). Comparing extra-retinal information about distance and direction. Vision Research, 40, 1649-1651.

Burge, J., Girshick, A. R., \& Banks, M. S. (2010). Visual-haptic adaptation is determined by relative reliability. Journal of Neuroscience, 30, 7714-7721.

Coello, Y., Danckert, J., Blangero, A., \& Rossetti, Y. (2007). Do visual illusions probe the visual brain? Illusions in action without a dorsal visual stream. Neuropsychologia, 45, 1849-1858.

Cohen, H. B. (1966). Some critical factors in prism adaptation. The American Journal of Psychology, 79, 285-290.

Collewijn, H., \& Erkelens, C. J. (1990). Binocular eye movements and the perception of depth. In E. Kowler (Ed.), Eye movements and their role in visual and cognitive processes (pp. 213-261). Amsterdam: Elsevier.

Craske, B. (1966). Change in transfer function of joint receptor output. Nature, 210, 764-765.

Craske, B. (1967). Adaptation to prisms: change in internally registered eye-position. British Journal of Psychology, 58, 329-335.

Craske, B., \& Crawshaw, M. (1974). Adaptive changes of opposite sign in the oculomotor systems of the two eyes. The Quarterly Journal of Experimental Psychology, 26, 106-113.

Craske, B., \& Crawshaw, M. (1975). Oculomotor adaptation to prisms is not simply a muscle potentiation effect. Perception \& Psychophysics, 18, 105-106.

Craske, B., \& Crawshaw, M. (1978). Spatial discordance is a sufficient condition for oculomotor adaptation to prisms: eye muscle potentiation need not be a factor. Perception \& Psychophysics, 23, 75-79.

Cressman, E. K., \& Henriques, D. Y. (2009). Sensory recalibration of hand position following visuomotor adaptation. Journal of Neurophysiology, 102, 3505-3518.

Cumming, B. G., \& Judge, S. J. (1986). Disparity-induced and blur-induced convergence eye movement and accommodation in the monkey. Journal of Neurophysiology, 55, 896-914.

Danckert, J., Goldberg, L., \& Broderick, C. (2009). Damage to superior parietal cortex impairs pointing in the sagittal plane. Experimental Brain Research, 195, 183-191.

Day, R. H., \& Singer, G. (1967). Sensory adaptation and behavioral compensation with spatially transformed vision and hearing. Psychological Bulletin, 67, 307-322.

Donaldson, I. M. (2000). The functions of the proprioceptors of the eye muscles. Philosophical Transactions of the Royal Society of London. Series B, Biological Sciences, 355, 1685-1754.

Ebenholtz, S. M. (1974). The possible role of eye-muscle potentiation in several forms of prism adaptation. Perception, 3, 477-485.

Ebenholtz, S. M. (1981). Hysteresis effects in the vergence control system: perceptual implications. In D. F. Fisher, R. A. Monty \& J. W. Senders (Eds.), Eye movements: visual perception and cognition (pp. 83-94). Hillsdale, NJ: Erlbaum.

Ebenholtz, S. M., \& Fisher, S. K. (1982). Distance adaptation depends upon plasticity in the oculomotor control system. Perception \& Psychophysics, 31, 551-560.

Ebenholtz, S. M., \& Wolfson, D. M. (1975). Perceptual aftereffects of sustained convergence. Perception \& Psychophysics, 17, 485-491.

Epstein, W. (1975). Recalibration by pairing: a process of perceptual learning. Perception, 4, 59-72.

Ernst, M. O., \& Bülthoff, H. H. (2004). Merging the senses into a robust percept. Trends in Cognitive Sciences, 8, 162-169. 
Ferraina, S., Battaglia-Mayer, A., Genovesio, A., Archambault, P., \& Caminiti, R. (2009). Parietal encoding of action in depth. Neuropsychologia, 47, 1409-1420.

Fisher, S. K., \& Ciuffreda, K. J. (1990). Adaptation to optically-increased interocular separation under naturalistic viewing conditions. Perception, 19, 171-180.

Fisher, S. K., \& Ebenholtz, S. M. (1986). Does perceptual adaptation to telestereoscopically enhanced depth depend on the recalibration of binocular disparity? Perception \& Psychophysics, 40, 101-109.

Galletti, C., Fattori, P., Gamberini, M., \& Kutz, D. F. (1999). The cortical visual area V6: brain location and visual topography. European Journal of Neuroscience, 11, 3922-3936.

Gauthier, G. M., Nommay, D., \& Vercher, J. L. (1990a). Ocular muscle proprioception and visual localization of targets in man. Brain, 113, 1857-1871.

Gauthier, G. M., Nommay, D., \& Vercher, J. L. (1990b). The role of ocular muscle proprioception in visual localization of targets. Science, 249, 58-61.

Ghahramani, Z., Wolpert, D. M., \& Jordan, M. I. (1997). Computational models for sensorimotor integration. In P. G. Morasso \& V. Sanguineti (Eds.), Self-organization, computational maps and motor control (pp. 117-147). Amsterdam: Elsevier.

Hamilton, C. R. (1964). Intermanual transfer of adaptation to prisms. The American Journal of Psychology, 77, 457-462.

Harris, C. S. (1963). Adaptation to displaced vision: visual, motor, or proprioceptive change? Science, 140, 812-813.

Harris, C. S. (1965). Perceptual adaptation to inverted, reversed, and displaced vision. Psychological Review, 72, 419-444.

Hatada, Y., Miall, R. C., \& Rossetti, Y. (2006). Long lasting aftereffect of a single prism adaptation: Directionally biased shift in proprioception and late onset shift of internal egocentric reference frame. Experimental Brain Research, 174, 189-198.

Hay, J. C., \& Pick, H. L., Jr. (1966). Visual and proprioceptive adaptation to optical displacement of the visual stimulus. Journal of Experimental Psychology, 71, 150-158.

Held, R., \& Hein, A. (1958). Adaptation to disarranged hand-eye coordination contingent upon reafferent stimulation. Perceptual and Motor Skills, 8, 87-90.

Helmholtz, H., von. (1910). Handbuch der Physiologischen Optik. 3rd edition. Voss, Leipzig. English translation by JPC Southall (1925) Treatise on physiological optics (Vol. 3. The perceptions of vision). Rochester, NY: Optical Society of America.

Howard, I. P. (1970). The adaptability of the visual-motor system. In K. J. Connolly (Ed.), Mechanisms of motor skill development. London: Academic Press.

Howard, I. P. (1971). Perceptual learning and adaptation. British Medical Bulletin, 27, 248-252.

Howard, I. P., \& Rogers, B. J. (2002). Seeing in depth (Vol. 2. Depth perception). Toronto: I. Porteous.

Judge, S. J., \& Bradford, C. M. (1988). Adaptation to telestereoscopic viewing measured by onehanded ball-catching performance. Perception, 17, 783-802.

Judge, S. J., \& Miles, F. A. (1985). Changes in the coupling between accommodation and vergence eye movements induced in human subjects by altering the effective interocular separation. Perception, 14, 617-629.

Kalil, R. E., \& Freedman, S. J. (1966). Persistence of ocular rotation following compensation for displaced vision. Perceptual and Motor Skills, 22, 135-139.

Kitazawa, S., Kimura, T., \& Uka, T. (1997). Prism adaptation of reaching movements: specificity for the velocity of reaching. Journal of Neuroscience, 17, 1481-1492.

Kitazawa, S., Kohno, T., \& Uka, T. (1995). Effects of delayed visual information on the rate and amount of prism adaptation in the human. Journal of Neuroscience, 15, 7644-7652.

Kornheiser, A. S. (1976). Adaptation to laterally displaced vision: a review. Psychological Bulletin, 83, 783-816.

Landy, L. S., Maloney, L. T., Johnston, E. B., \& Young, M. (1995). Measurement and modeling of depth cue combination: in defense of weak fusion. Vision Research, 35, 389-412.

Lester, G. (1968). The case for efferent change during prism adaptation. The Journal of Psychology, $68,9-13$. 
Luauté, J., Schwartz, S., Rossetti, Y., Spiridon, M., Rode, G., Boisson, D., \& Vuilleumier, P. (2009). Dynamic changes in brain activity during prism adaptation. Journal of Neuroscience, 29, 169178.

Magescas, F., \& Prablanc, C. (2006). Automatic drive of limb motor plasticity. Journal of Cognitive Neuroscience, 18, 75-83.

Mamassian, P., Landy, M. S., \& Maloney, L. T. (2002). Bayesian modelling of visual perception. In R. P. N. Rao, B. A. Olshausen \& M. S. Lewicki (Eds.), Probabilistic models of the brain: perception and neural function (pp. 13-36). Cambridge, MA: MIT Press.

Martin, T. A., Keating, J. G., Goodkin, H. P., Bastian, A. J., \& Thach, W. T. (1996a). Throwing while looking through prisms. I. Focal olivocerebellar lesions impair adaptation. Brain, 119, 11831198.

Martin, T. A., Keating, J. G., Goodkin, H. P., Bastian, A. J., \& Thach, W. T. (1996b). Throwing while looking through prisms. II. Specificity and storage of multiple gaze-throw calibrations. Brain, 119 (Pt 4), 1199-1211.

McLaughlin, S. C., \& Bower, J. L. (1965). Selective intermanual transfer of adaptive effects during adaptation to prism. Psychonomic Science, 3, 69-70.

McLaughlin, S. C., Rifkin, K. I., \& Webster, R. G. (1966). Oculomotor adaptation to wedge prisms with no part of the body seen. Perception \& Psychophysics, 1, 452-458.

Mon-Williams, M., \& Tresilian, J. R. (1999). Some recent studies on the extraretinal contribution to distance perception. Perception, 28, 167-181.

Mon-Williams, M., Wann, J. P., Jenkinson, M., \& Rushton, K. (1997). Synaesthesia in the normal limb. Proceedings: Biological Sciences, 264, 1007-1010.

Morley, J. W., Judge, S. J., \& Lindsey, J. W. (1992). Role of monkey midbrain near-response neurons in phoria adaptation. Journal of Neurophysiology, 67, 1475-1492.

Morton, S. M., \& Bastian, A. J. (2004). Prism adaptation during walking generalizes to reaching and requires the cerebellum. Journal of Neurophysiology, 92, 2497-2509.

Owens, D. A., \& Leibowitz, H. W. (1980). Accommodation, convergence, and distance perception in low illumination. American Journal of Optometry and Physiological Optics, 57, 540-550.

Paap, K. R., \& Ebenholtz, S. M. (1977). Concomitant direction and distance aftereffects of sustained convergence: a muscle potentiation explanation for eye-specific adaptation. Perception \& Psychophysics, 21, 307-314.

Plooy, A., Tresilian, J. R., Mon-Williams, M., \& Wann, J. P. (1998). The contribution of vision and proprioception to judgements of finger proximity. Experimental Brain Research, 118, 415420.

Prablanc, C., Tzavaras, A., \& Jeannerod, M. (1975). Adaptation of the two arms to opposite prism displacements. The Quarterly Journal of Experimental Psychology, 27, 667-671.

Prevosto, V., Graf, W., \& Ugolini, G. (2009). Cerebellar inputs to intraparietal cortex areas LIP and MIP: functional frameworks for adaptive control of eye movements, reaching, and arm/eye/head movement coordination. Cerebral Cortex, 20, 214-228.

Priot, A. E., Laboissière, R., Sillan, O., Roumes, C., \& Prablanc, C. (2010). Adaptation of egocentric distance perception under telestereoscopic viewing within reaching space. Experimental Brain Research, 202, 825-836.

Quinlan, D. J., \& Culham, J. C. (2007). fMRI reveals a preference for near viewing in the human parieto-occipital cortex. Neuroimage, 36, 167-187.

Redding, G. M., Rossetti, Y., \& Wallace, B. (2005). Applications of prism adaptation: a tutorial in theory and method. Neuroscience and Biobehavioral Reviews, 29, 431-444.

Redding, G. M., \& Wallace, B. (1992). Adaptive eye-hand coordination: implication of prism adaptation for perceptual-motor organization. In L. Proteau \& D. Elliott (Eds.), Vision and motor control (pp. 105-127). Amsterdam: Elsevier Science Publishers.

Redding, G. M., \& Wallace, B. (2006). Prism adaptation and unilateral neglect: review and analysis. Neuropsychologia, 44, 1-20.

Sarlegna, F., Blouin, J., Bresciani, J. P., Bourdin, C., Vercher, J. L., \& Gauthier, G. M. (2003). Target and hand position information in the online control of goal-directed arm movements. Experimental Brain Research, 151, 524-535. 
Shebilske, W. L., Karmiohl, C. M., \& Proffitt, D. R. (1983). Induced esophoric shifts in eye convergence and illusory distance in reduced and structured viewing conditions. Journal of Experimental Psychology. Human Perception and Performance, 9, 270-277.

Simani, M. C., McGuire, L. M., \& Sabes, P. N. (2007). Visual-shift adaptation is composed of separable sensory and task-dependent effects. Journal of Neurophysiology, 98, 2827-2841.

Snijders, H. J., Holmes, N. P., \& Spence, C. (2007). Direction-dependent integration of vision and proprioception in reaching under the influence of the mirror illusion. Neuropsychologia, 45, 496-505.

Steinbach, M. J., \& Smith, D. R. (1981). Spatial localization after strabismus surgery: evidence for inflow. Science, 213, 1407-1409.

Templeton, W. B., Howard, I. P., \& Wilkinson, D. A. (1974). Additivity of components of prismatic adaptation. Perception \& Psychophysics, 15, 249-257.

van Beers, R. J., Wolpert, D. M., \& Haggard, P. (2002). When feeling is more important than seeing in sensorimotor adaptation. Current Biology, 12, 834-837.

von Hofsten, C. (1976). The role of convergence in visual space perception. Vision Research, 16, 193198.

von Hofsten, C. (1979). Recalibration of the convergence system. Perception, 8, 37-42.

Wallace, B., \& Redding, G. M. (1979). Additivity in prism adaptation as manifested in intermanual and interocular transfer. Perception \& Psychophysics, 25, 133-136.

Wallach, H., \& Frey, K. (1972). Adaptation in distance perception based on oculomotor cues. Perception \& Psychophysics, 11, 77-83.

Wallach, H., Frey, K., \& Bode, K. (1972). The nature of adaptation in distance perception based on oculomotor cues. Perception \& Psychophysics, 11, 110-116.

Wallach, H., \& Smith, A. (1972). Visual and proprioceptive adaptation to altered oculomotor adjustments. Perception \& Psychophysics, 11, 413-416.

Weiner, M. J., Hallett, M., \& Funkenstein, H. H. (1983). Adaptation to lateral displacement of vision in patients with lesions of the central nervous system. Neurology, 33, 766-772.

Welch, R. B. (1969). Adaptation to prism-displaced vision: the importance of target pointing. Perception \& Psychophysics, 5, 305-309.

Welch, R. B. (1974). Speculations on a model of prism adaptation. Perception, 3, 451-460.

Welch, R. B. (1986). Adaptation of space perception. In K. R. Boff, L. Kaufman \& J. P. Thomas (Eds.), Handbook of perception and human performance (Vol. 1. Sensory process and perception). New York: Wiley.

Welch, R. B., Choe, C. S., \& Heinrich, D. R. (1974). Evidence for a three-component model of prism adaptation. Journal of Experimental Psychology, 103, 700-705.

Wilkinson, D. A. (1971). Visual-motor control loop: a linear system? Journal of Experimental Psychology, 89, 250-257. 


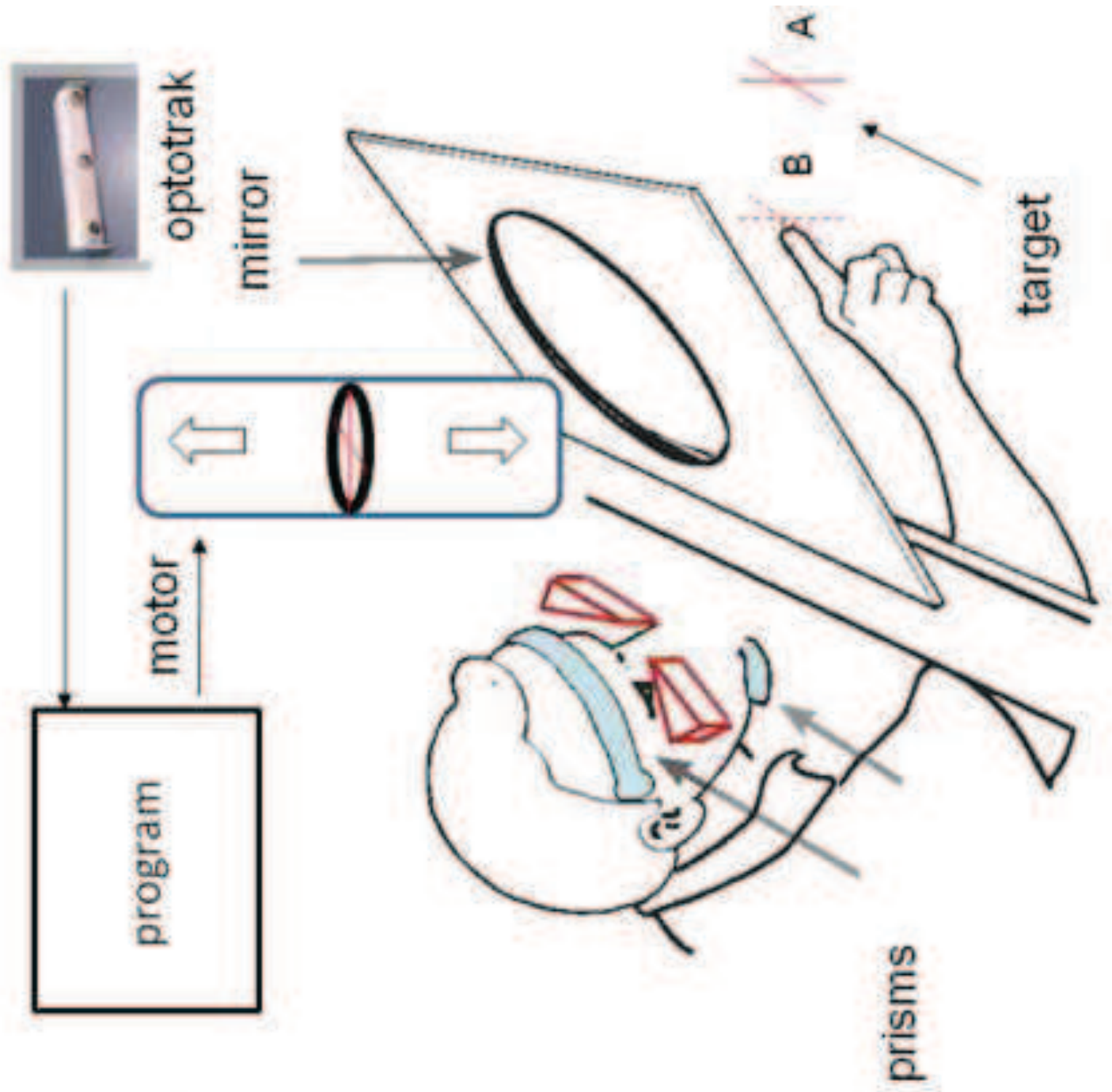

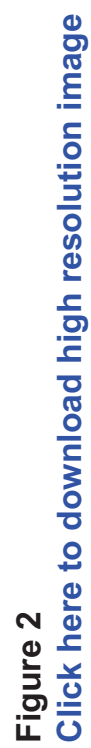


Figure 3

Click here to download high resolution image

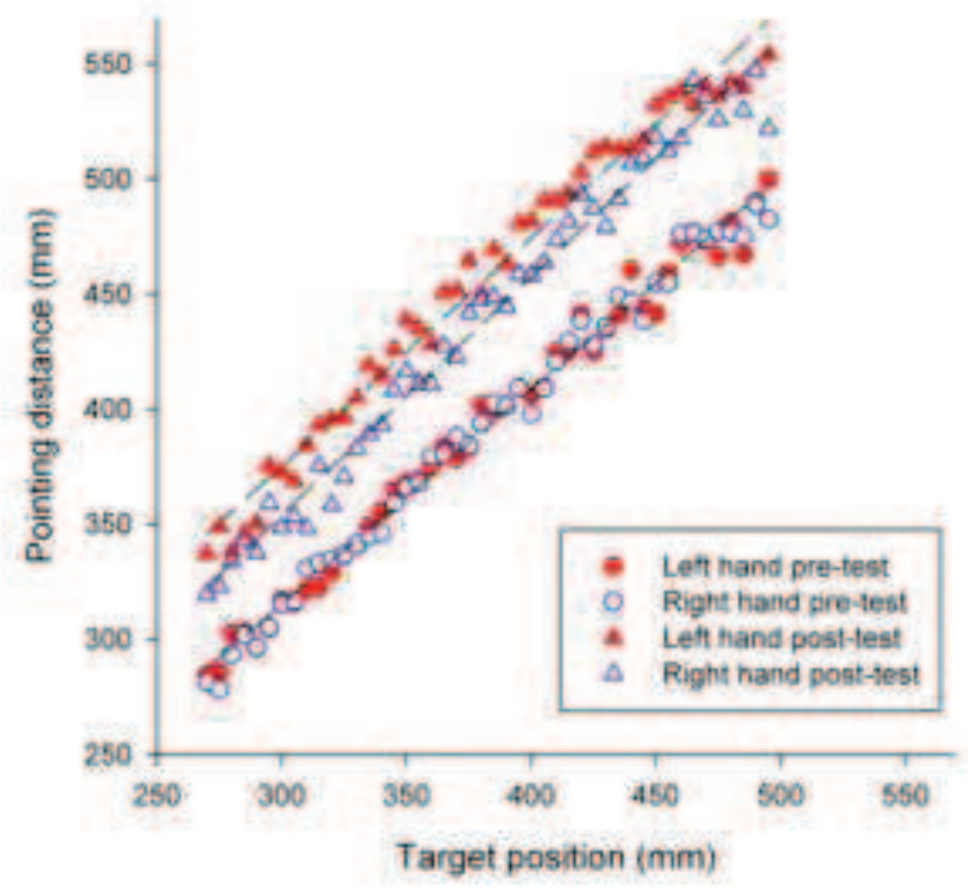


Figure 4

Click here to download high resolution image

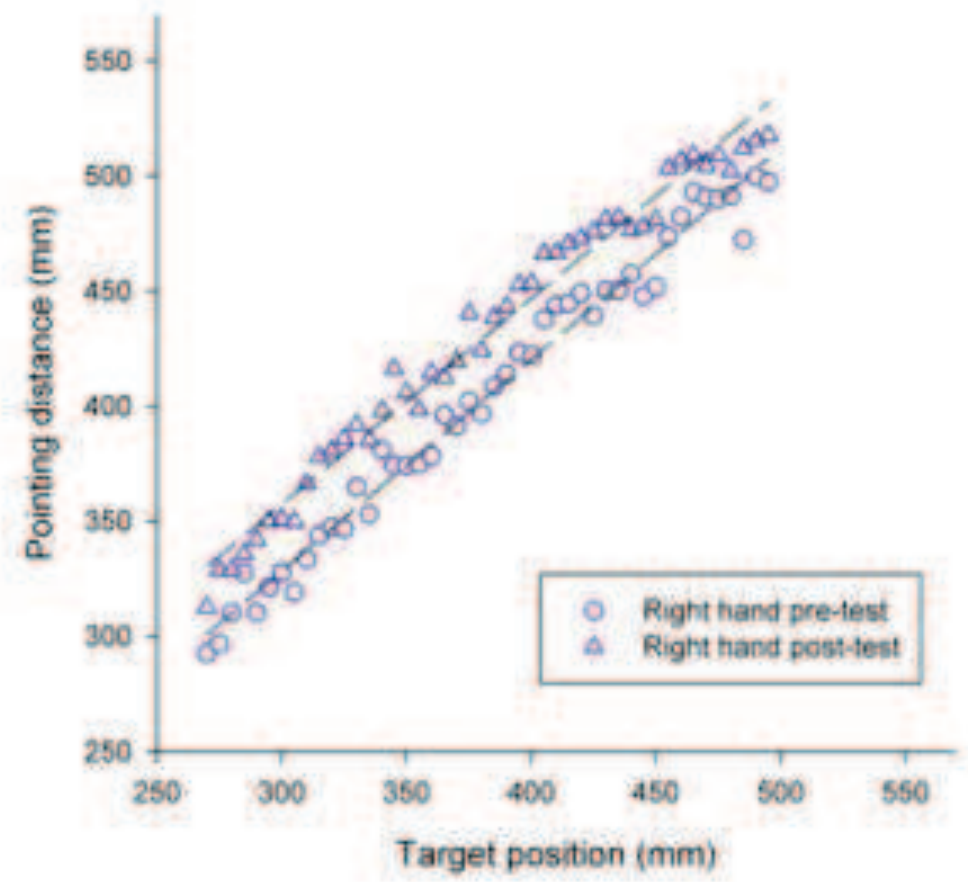




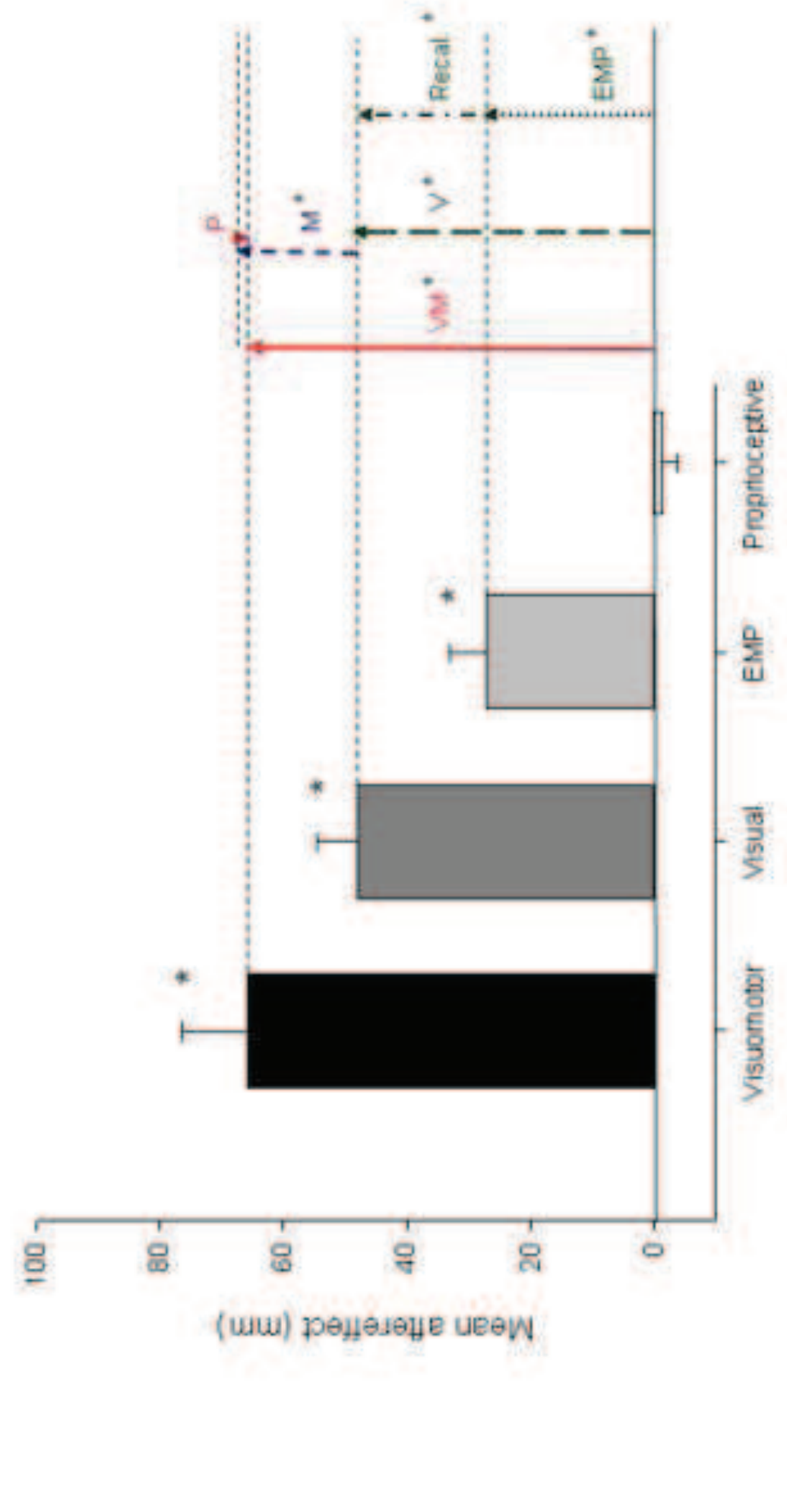

